\title{
Alpha Spectroscopy and X-ray Induced Photocurrent Studies of a SC CVD Diamond Detector fabricated with PLD Contacts
}

\author{
Mohamed A.E. Abdel-Rahman ${ }^{* 1,2}$, Annika Lohstroh ${ }^{2}$, Peter Bryant ${ }^{2}$ \\ ${ }^{1}$ Nuclear Engineering Department, Military Technical College, Kobry El-Kobba, Cairo, Egypt \\ ${ }^{2}$ Department of Physics, University of Surrey, Guildford, Surrey GU2 7XH, United Kingdom
}

\begin{abstract}
Using amorphous Carbon blended with $\mathrm{Nickel}(\mathrm{C} / \mathrm{Ni})$ as electrodes for a polycrystalline diamond radiation detector $(\mathrm{PC}-\mathrm{C} / \mathrm{Ni})$ was demonstrated previously as a novel technique to produce near-tissue equivalent X-ray dosimeters. In this project, we introduce the first characterisation of a (Chemical Vapour Deposited) single crystal diamond sandwich detector (with thickness of $0.4 \mathrm{~mm}$ ) fabricated with this technique, labelled SC-C/Ni. To examine the performance of pulsed laser deposition (PLD) $\mathrm{C} / \mathrm{Ni}$ as an electrode, alpha spectroscopy measurements and X-ray induced photocurrent measurements were studied as a function of the applied bias at room temperature and compared with those of the aforementioned PC-C/Ni.

The alpha particle spectroscopy measurement data allows us to differentiate between electron and hole contributions to the charge transport signal, whilst the X-ray data was investigated in terms of dose rate-linearity, sensitivity, signal to noise ratio (SNR) and photoconductive gain.

In the case of electron sensitive alpha induced signals, a Charge Collection Efficiency (CCE) higher than $90 \%$ has been observed at a bias of $-40 \mathrm{~V}$ and $100 \% \mathrm{CCE}$ at $-300 \mathrm{~V}$, with energy resolution of $\sim 3 \%$ for $5.48 \mathrm{MeV}$ alpha particles. The sample showed very poor spectroscopy performance for hole sensitive signals up to $200 \mathrm{~V}$.

The X-ray induced photocurrents show a high SNR of $\sim 7.3 \times 10^{3}$, an approximately linear relationship between the photocurrent versus dose rate and a sensitivity of $4.87 \mu \mathrm{C} / \mathrm{Gy} \cdot \mathrm{mm}^{3}$. The photoconductive gain of SC-C/Ni is calculated to be $\sim 20$, this gain calculation might be explained by trapping effects as investigated in the alpha spectroscopy measurements.
\end{abstract}

Keywords: CVD diamond based radiation detector, alpha spectroscopy, dosimetric properties, carbon electrode, PLD

\section{Introduction}

Diamond is an important material for radiation detection purposes especially when used in the field of medical applications. One of its advantages mostly is its near tissue equivalence $[1,2]$ where its atomic number can be compared with that of human tissue ( $\mathrm{Z}=6$ for diamond and $\mathrm{Z} \approx 7.5$ for human tissues). Thus the energy absorbed by diamond based radiation detectors is comparable to that absorbed by human tissue, consequently reduces the need for correction procedures required by other semiconductor materials [3]. Diamond's high bandgap $(\sim 5.5 \mathrm{eV})[4,5]$ results in a low leakage current and hence low dark noise. Transient current technique (TCT) studies in literature show that electronic grade chemical vapor deposited (CVD) SC diamond has high zero field electron and hole drift mobilities [6-8], which permit a fast time response of the signal. TCT data probes electron and hole drift velocities, charge carrier lifetimes, as well as effective concentrations of space charge in the detector bulk [9]. However, there are not many studies which present a comparison between the spectroscopic performance of SC CVD charge particle detectors and electrode fabrication method [10].

It is advisable to use electrodes with low thickness and low $\mathrm{Z}$ in medical field applications. For this reason, carbon is considered the optimum choice as electrical electrode. Pulsed Laser Deposition (PLD) of amorphous carbon and/or carbon/metal composition allows controlling the characteristics of the electrode by changing the deposition parameters [11]. Other advantageous properties of diamond in this context is radiation and mechanical hardness, high thermal conductivity, non-toxicity and chemical inertness [6]. Amorphous carbon films (containing a high

* Corresponding author: e-mail: mabdelrahman@mtc.edu.eg , Phone: (+2) 01222431122 
ratio of $\mathrm{sp}^{3}$ bonds) are called diamond-like carbon (DLC) as they have similar properties to diamond. Thus the evolution of DLC as electrode films could help applications especially in medical X-ray dosimetry. Therefore a SC-C/Ni sample with a mixed C/Ni electrode was fabricated using the PLD technique [11]. Spectroscopic and Xray induced photocurrent data was obtained and a comparisons drawn to literature. The presented study is focused on understanding the effect of using a symmetric $\mathrm{C} / \mathrm{Ni}$ electrode sandwich structured radiation detector, in order to evaluate this electrode fabrication method as a future candidate for medical dosimetry devices. Hence low $\mathrm{Z} /$ Carbon based electrodes are favoured in order to maintain the tissue equivalence in future devices. Alpha particle spectroscopy was used to study the electron and hole transport properties of the SC-C/Ni device complementing the results of the X-ray measurements.

\section{Experimental Details}

\subsection{Device Fabrication and Mounting}

According to the previous reported advantages by Abdel-Rahman et al. [12] of a polycrystalline diamond samples with $\mathrm{C} / \mathrm{Ni}$ electrodes, a single crystal sample with the same electrical electrode was fabricated labelled SC-C/Ni. The sample was an electronic grade Chemical vapour deposited diamond procured from Element Six Ltd.; nominal substitutional nitrogen and boron concentrations are $<1 \mathrm{ppm}$ and $<0.05 \mathrm{ppm}$ in this material. The description of the sample fabrication has been issued previously and can be found in detail in [12]. The diamond sample base (with dimensions $4 \times 4 \times 0.4 \mathrm{~mm}^{3}$ ) was metalized using $\mathrm{C} / \mathrm{Ni}$ with a square of $2 \times 2 \mathrm{~mm}^{2}$. The electrical electrode, made of $\mathrm{C} / \mathrm{Ni}$ on diamond, with thickness of $80 \mathrm{~nm}$ was deposited using PLD in a sandwich geometry as described in [12]. Prior to the metallization, the base crystal was chemically treated by cleaning and oxidation of the material surface before any deposition to promote the diamond surface properties and ensure an oxygen terminated surface [13].

The target material used for the deposition process was $\mathrm{C} / \mathrm{Ni}$ : 80:20 (at. \%). The $\mathrm{C} / \mathrm{Ni}$ was deposited on the centre of the sample on both sides using a shadow mask with dimensions $2 \times 2 \mathrm{~mm}^{2}$. Ni is a strong and efficient $\mathrm{sp}^{2}$ carbon catalyst on diamond, which makes it etch the diamond surface. The whole procedure process is repeated for the second side of the crystal. The deposition parameters chosen are expected to result in a $\mathrm{C} / \mathrm{Ni}$ (at. \%) ratio of approx. (49:51) and the D to G peak ratio in Raman spectra observed with $514 \mathrm{~nm}$ laser excitation after annealing is expected to be $0.8[11,14]$. Only one single crystal sample was fabricated, however - similar work on polycrystalline material, where multiple samples deposited under the same conditions, showed consistent results in the Raman and X-ray photocurrent measurements [14].

The sample was carefully mounted on a printed circuit board (PCB), using a small dot of Colloidal Graphite Epoxy on the centre of the deposited square to avoid epoxy spilling over the electrode edges. There is no guard ring and hence the electric field will extend beyond the contact area and irradiation hitting the sample near the contact edges will contribute to the signal. A gold wire (thickness of $24 \mu \mathrm{m}$ ) bond is fixed on the top contact of the sample by using Conductive Epoxy. The other end is connected to the PCB utilizing silver paint. The pads will be coupled with electrical wires that are joined to a BNC connector.

\subsection{X-ray Induced photocurrent measurements}

The broad beam X-ray set-up used to measure the dosimetric properties of the diamond device was performed in air using an Oxford instruments XF5011 $50 \mathrm{kVp}$ X-ray tube with a Mo target; The Mo $\mathrm{K}_{\alpha}$ line energy is $17.48 \mathrm{keV}$ and the majority of the bremsstrahlung intensity can also be found above that energy when the tube is operated at $50 \mathrm{kVp}$ (which is the case in our experiments). At $17.48 \mathrm{keV}, 94 \%$ of the X-ray photons will be transmitted through the $0.4 \mathrm{~mm}$ thick sample - this value will increase even further for higher X-ray photon energies [15]. 
Hence, the energy deposited throughout the thickness of the diamond sample by the X-ray irradiation is expected to be almost uniform. The photocurrent data were obtained at different dose rates. The dose rate range used was achieved by varying the X-ray tube anode current up to a maximum value of $1 \mathrm{~mA}$; the sample was mounted at $11.5 \mathrm{~cm}$ distance from the X-ray tube exit port. The quoted dose rate values correspond to the air kerma measured at the sample position using a $0.6 \mathrm{~cm}^{3}$ ionization chamber, model number NE2571A. During the calibration process the diamond sample is replaced with the ionization chamber. This chamber is connected to a Standard Farmer Ionisation Chamber readout unit with an integrated voltage source and dose rate meter (NE technology LTD, UK) via a RS 232 Port. This gives an approximation of the energy deposited in the samples neglecting differences due to air ionisation, scattering and absorption effects.

All photocurrent measurements were carried out by using a Keithley 487 voltage source and picoammeter. In general, when the detector is exposed to irradiation, an electrical current $\left(I_{g e n}\right)$ will be generated in the detector, which sequentially induces a current $\left(I_{\text {measured }}\right)$ in the external circuit. The photoconductive gain value is defined as the ratio between the induced photocurrent measured by the electrometer and the theoretical value calculated within the detector as a result of irradiation, i.e. dose rate. The estimated theoretical current can be calculated from the equation [13]:

$$
I_{\text {generated }}=\frac{D \rho e v}{w}
$$

Where: $D, \rho, e, v$ and $w$ are the dose rate, density of diamond, elementary charge, sensitive volume and the average energy required to produce an electron hole pair (e-h pair) in diamond (13.2 eV) [2] respectively.

Table (1) shows both the generated current within the crystal and the measured current and from these two values the detector gain is calculated. All these measurements were performed in air at a dose rate and a bias voltage of $1.5 \mathrm{~Gy} / \mathrm{min}$ and $50 \mathrm{~V}$, respectively.

A preliminary study of the linearity of the photocurrent over a narrow range of dose rates was carried out by varying the X-ray tube anode current. The variance of the current $I$ versus the irradiated dose rate $(D)$, can be fitted using the Fowler relationship [7]:

$$
I=I_{\text {dark }}+R D^{\Delta}
$$

Where: $I_{d a r k}$ is the dark (leakage) current and $R$ is a fitting parameter. The exponent $\Delta$, referred to as the fitting coefficient, or the linearity index is a constant showing the deviation from linearity. It is expected to vary between 0.5 and 1.0 if all the traps in the crystal have the same capture cross section [17]. Moreover, it is important to acknowledge that this bulk interpretation of $\Delta$ is not always valid, as our earlier research [12] shows that the contact fabrication and annealing can drastically change the measured value of delta $(\Delta)$, although it is unlikely that the process significantly alters the bulk properties of the material.

Measuring $\Delta$ is regarded as suitable tool for x-ray dosimetry purposes, as it determines a correction factor used to calculate the absorbed dose. In case $\Delta=1$, the integrated induced current for the diamond sample does not depend on the dose rate making it optimum for use in dosimetry applications.

\subsection{Alpha Spectroscopy}

The alpha response of the diamond device was recorded to estimate the energy resolution, charge collection efficiency $(C C E)$ and mobility-lifetime $(\mu \tau)$ product. This was acquired by using a $3.5 \mathrm{kBq}{ }^{241} \mathrm{Am}$ alpha source with energy of $5.48 \mathrm{MeV}$.

The diamond sample was irradiated under vacuum with alpha particles from the ${ }^{241}$ Am source, which was mounted at distances between $0.5 \mathrm{~mm}$ and $15 \mathrm{~mm}$ from the sample surface. These measurements were carried out in the 
dark, at room temperature with pressure less than $10^{-1}$ mbar. The benefit of performing these measurements in a vacuum is to avoid the loss of energy from the alpha particles due to attenuation in air. The device was connected to a charge sensitive preamplifier (ORTEC 142A) and the output signal coupled to a shaping amplifier (ORTEC 570), followed by a multi-channel analyser (MCA, DSP-spect). During the irradiation with alpha particles, a high voltage was applied to the detector via the pre-amplifier.

Whilst performing the energy calibration the pre-amplifier is disconnected from the detector and directly connected to a 2.31 pf capacitor. This allows an absolute system calibration of the electronics in the system to be performed.

The range of a $5.48 \mathrm{MeV} \alpha$-particles within diamond is of the order of $15 \mu \mathrm{m}$ [8] (and as such very adjacent to the surface of the detector). Approximating the ${ }^{241} \mathrm{Am}$ source as a point source and making the simplifying assumption that the alpha particle energy is deposited uniformly throughout the first $15 \mu \mathrm{m}$ depth, the maximum dose rate caused by the alpha particles at $5 \mathrm{~mm}$ distance can be estimated to be $<1 \mathrm{cGy} / \mathrm{min}$. Similarly, the $\gamma$ dose rate incident on the sample from the ${ }^{241} \mathrm{Am}$ source at that distance is approximately $0.36 \mathrm{cGy} / \mathrm{min}$ [19]. For this cause when irradiating the surface of the detector where a positive bias is applied, the majority of the signal created within the device will be from holes. This is due to the e-h pairs being produced near to the anode and as such the holes travel though almost the entire thickness of the device till they reach the cathode whereas the electrons move through only a small distance of the device till they reach the anode. It is therefore possible to study the characteristics of electrons and holes separately by changing the applied bias voltage to the irradiated surface. Assuming a constant electric field throughout the device and no re-emission of trapped charge carriers within the integration time, the $C C E$ of a radiation detector can be calculated by using the Hecht equation [20]:

$$
C C E=\frac{\mu \tau V}{d^{2}}\left[1-\exp \left(\frac{-d^{2}}{\mu \tau V}\right)\right]
$$

where $V$ is the detector bias and $d$ is the detector thickness.

\section{Results and Discussion}

\subsection{Alpha Spectroscopy}

Figure (1) shows the spectra of the ${ }^{241} \mathrm{Am}$ of $5.48 \mathrm{MeV} \alpha$-particles $(3.5 \mathrm{kBq}$ ) acquired with the SC-C/Ni diamond sample at different bias voltages (negative and positive bias, drift of electrons and holes, respectively) ranging from 50 to $200 \mathrm{~V}$. With an increase in bias voltage the peak centroid shifts to a higher energy position. The electron sensitive peak (at negative bias) exhibits a low energy shoulder, which is more obvious than those reported in literature for similar measurements [7-9]. This may be caused by using an uncollimated source and the lack of a guard ring, producing some lower energy counts when the alpha particles interact with the bare diamond near the electrode edges, where the electric field is weaker. Because of the asymmetry in the shape of the peak, the results of a Gaussian fit (used to determine the centroid location \& FWHM) are sensitive to the selection of the region of interest (ROI) for the fitting. In this particular case various ranges were chosen for the ROI and it was found that the variation in both the centroid position and FWHM are $\sim 0.5 \%$. A FWHM of $160 \mathrm{keV}\left(\Delta \mathrm{E} / \mathrm{E}_{\alpha} \approx 3 \%\right)$ was recorded for negative bias (for electron transport) of $-150 \mathrm{~V}$. The measured values of FWHM, at negative bias, are consistent with the typical value $(\sim 3 \%)$ suggested by Galbiati et al [21] for a SC diamond having DLC/Pt/Au contacts when exposed to a ${ }^{241} \mathrm{Am} 5.48 \mathrm{MeV}$ alpha source.

At positive bias (hole transport) the behaviour of the sample is clearly different, the centroid peak position is much less than that of an equivalent negative bias. The $C C E$ has not yet reached the plateau level even at $300 \mathrm{~V}$. The FWHM value is higher than its corresponding value at the -ve bias and it reaches a value of around $400 \mathrm{keV}$ $\left(\Delta \mathrm{E} / \mathrm{E}_{\alpha} \approx 8.5 \%\right)$ at $150 \mathrm{~V}$. 

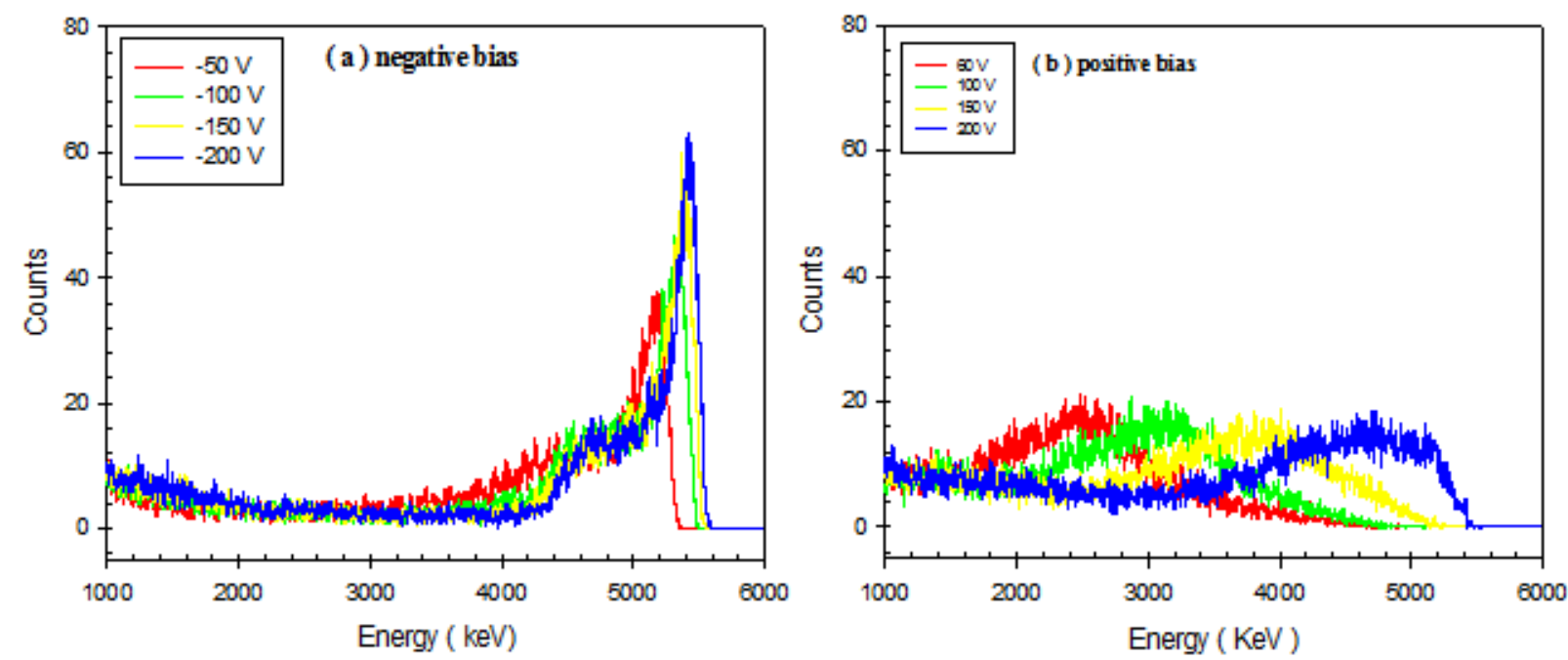

Figure (1): Alpha spectra in SC-C/Ni diamond sample under (a) negative and (b) positive bias.

The electron sensitive signals for $\mathrm{SC}-\mathrm{C} / \mathrm{Ni}$ are observed as superior compared to those produced predominantly by holes. It should be noted that the measurements were repeated twice to examine the reproducibility of the measurements. The repeat measurements showed reasonable reproducibility for this sample. This is in contrast to several reports that have obviously identified that hole drift velocities are superior than electron drift velocities in electronic grade diamond and typically CCE spectra in the literature are high quality at both polarities [7-9]. Hence, it is unlikely that the poor quality of the hole sensitive signals is produced by the quality of the bulk material of the device. A possible explanation for the poor hole performance in our sample of single-crystal CVD diamond could be trapping at the diamond/electrode interface, rather than in the diamond bulk.

Figure (2) shows the obtained CCE and the corresponding Hecht equation fit for both electrons (negative bias) and holes (positive bias).

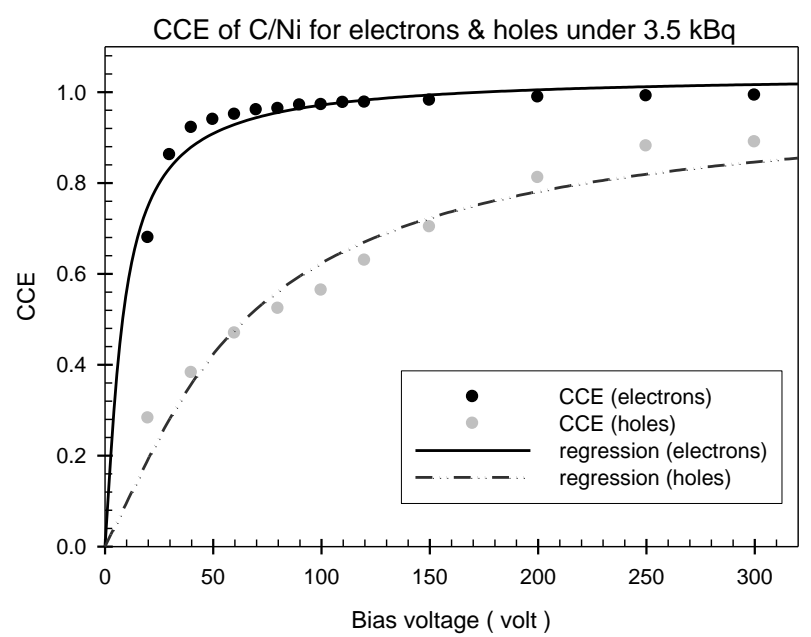

Figure (2): The $C C E$ and the Hecht plot of SC-C/Ni diamond sample as a function of both negative and positive bias.

It is clear from figure (2) that with a negative applied bias (electron drift) high CCEs $>90 \%$ are observed from -40 $\mathrm{V}$, increasing to a max. $C C E$ of $\sim 100 \%$, yielding an apparent high mobility-lifetime $\left(\mu_{e} \tau_{e}\right)$ product of $\left(\sim 1.16 \times 10^{-4}\right.$ $\pm 3.4 \times 10^{-5} \mathrm{~cm}^{2} \cdot \mathrm{V}^{-1}$ ). For positive bias (hole drift) the mean $C C E$ increases with bias reaching a maximum $C C E$ value of $\sim 89 \%$. Subsequently, the apparent mobility-lifetime $\left(\mu_{h} \tau_{h}\right)$ product extracted by fitting the Hecht equation yields a lower value (of $\sim 1.55 \times 10^{-5} \pm 1.1 \times 10^{-6} \mathrm{~cm}^{2} . \mathrm{V}^{-1}$ ). However, if the hole performance is predominantly compromised by trapping at the diamond/electrode interface (potentially also causing local polarisation, then the 
conditions for applying the Hecht equation are not fulfilled and the interpretation of the numerically extracted $\mu_{h} \tau_{h}$ value as a bulk property not applicable. It is possible that if the C/Ni electrode/interface has a high trap density for one charge carrier type that under positive applied bias the electric field is sufficiently reduced by space charge caused by filling these interface traps that the effective field the holes experience is too low to allow for good spectroscopy.

\subsection{X-ray measurements}

Figure (3) shows the linearity of the photocurrent as a function of the dose rate for the SC-C/Ni device. The dose rates are varied from 0.1 to $2.5(\mathrm{~Gy} / \mathrm{min})$ at a fixed bias of $50 \mathrm{~V}$, in order to allow comparisons to the previously published data in polycrystalline material. The studied dose rate range used in this work is not very wide and further developments of these devices will need to demonstrate similar performance over several magnitudes of dose rates.

An almost linear relationship was observed between the photocurrent and the dose rate for the SC-C/Ni device with a $\Delta$ value of $0.97 \pm 0.02$ obtained, as compared to the Poly-C/Ni $(\sim 0.86)$ [2] device. The comparison of the $\Delta$ values from the polycrystalline samples and the single crystal sample ( $\mathrm{SC}-\mathrm{C} / \mathrm{Ni}$ ) showed that $\Delta$ is changing according to the nature of the detector material. The obtained $\Delta$ value for the SC-C/Ni is consistent with the typical values reported by Descamps et al. [22] (0.98) for SC CVD diamond, with an electrical contact of $50 \mathrm{~nm}$ gold, using a linear accelerator with $6 \mathrm{MV}$ x-ray beam at varying dose rates from 1 to $6 \mathrm{~Gy} / \mathrm{min}$. Furthermore the measured values are consistent with De Angelis et al. [23] with value of 0.98 for PTW natural diamond using 6 MV photon beam at varying dose rates from 0.9 to $4.65 \mathrm{~Gy} / \mathrm{min}$ was measured at $100 \mathrm{~V}$. It was also observed that the obtained $\Delta$ value is in a good agreement with Hugtenburg et al. [20] results of $0.970 \pm 0.015$ and $0.975 \pm 0.003$ for commercially PTW natural diamond detector at both $45 \mathrm{kVp}$ and $100 \mathrm{kVp}$ X-ray photon beam over a dose range up to $8 \mathrm{~Gy}$, which represent a minimal sub-linearity of the dose-rate response. In another report, Nam et al. [5] investigated the effect of defect levels on $\Delta$ values, for synthetic CVD diamond materials, and the reliance of $\Delta$ values on the applied bias voltage, beam energy and type in the dosimetry of high-energy photon using 6 and 15 MV photon. Values of $\Delta$ ranging from $0.85-0.96$ were obtained for the photon beams for all the diamond detectors at $1 \mathrm{kV} / \mathrm{cm}$. These values were found to change with various defect levels present within the crystals. Also, the $\Delta$ values of crystals with high concentration of defect levels varied widely with the applied bias voltage.

Performing a linear regression on the data points obtained from the linearity curve of the diamond sample, as seen in figure (3), the value for the device sensitivity was calculated to be $\sim 7.8 \pm 0.27 \mu \mathrm{C} / \mathrm{Gy}$, corresponding to a specific sensitivity of $4.87 \pm 0.17 \mu \mathrm{C} / \mathrm{Gy} \cdot \mathrm{mm}^{3}$. This is a larger sensitivity compared to Poly-C/Ni, which has been obtained in a previous report [11] as $43.5 \pm 2.4 \mathrm{nC} / \mathrm{Gy} \cdot \mathrm{mm}^{3}$. This is attributed to the lower density of defects in the single crystal material leading to a higher signal currents than in polycrystalline samples [18]. A high specific sensitivity value was also reported by Cirrone et al. [6] with a value of $0.745 \pm 0.037 \mu \mathrm{C} / \mathrm{Gy} . \mathrm{mm}^{3}$ for commercial CVD diamond, grown by De Beers, using a $6 \mathrm{MV}$ photon beams. In another report, Nicholas Ade [7] found that for synthetic CVD diamond detectors, on exposure to mammography $\mathrm{x}$-rays in the energy range from 26 to $30 \mathrm{kVp}$, the sensitivity values ranging from 0.4 to $6.7 \mathrm{nC} / \mathrm{Gy} \cdot \mathrm{mm}^{3}$ where determined for the detectors varied with defect levels. This is believed that differences in crystal quality due to the presence and influence of impurity and defects would cause a discrepancy in the dosimetric performances of various diamond detectors.

The estimated gain value of the SC-C/Ni ( 20) is approximately 450 times greater than the typical value of Poly$\mathrm{C} / \mathrm{Ni}\left(\sim 44 \times 10^{-3}\right)$ as mentioned in a previous report [2]. The trapping may be able to take place at the electrode/diamond material interface, similar to the data shown by Schirru et al [6], which indicates a difference in X-ray signal with electrode fabrication process and [8] also shows a high sensitivity following annealing at 
temperatures that are not expected to change the bulk of the diamond. Photoconductive gain is expected to appear if the defect distribution in the device predominantly captures only one type of carrier (electron or hole), while the other type remains free $[16,8]$. When an electron-hole pair is generated and for example, holes are captured immediately in traps, then the corresponding electron remains free in the conduction band and contributes to the conductivity. When the electrons reach the anode and the cathode has ohmic properties (i.e. allows charge injection), then - on average - electrons will be injected at the cathode to replenish the negative charges that have exited at the anode. The injection is not necessarily instantaneous, i.e. it is likely to happen outside the integration time of the $\alpha$ spectroscopy system, which will only be sensitive to charges travelling within a few microseconds after the initial $\alpha$-particle has hit the detectors. In continuous current mode however, these injected electrons contribute to the signal until they recombine with the thermally de-trapped holes, thereby increasing the average effective lifetime of the electrons and thus increasing the photoconductive gain of the device, this process has been referred to as "sensitizing effect" in the literature $[16,6,8]$.

\begin{tabular}{cccccccccc}
\hline Device & $\begin{array}{c}\text { Bias } \\
\text { voltage } \\
\text { (V) }\end{array}$ & $\begin{array}{c}\text { Imeasured } \\
\text { (A) }\end{array}$ & $\begin{array}{c}\text { Standard } \\
\text { Deviation } \\
\text { (SD) }\end{array}$ & $\begin{array}{c}\text { Idark } \\
\text { (A) }\end{array}$ & Igenerated (A) & $\begin{array}{c}\text { Gain } \\
\text { factor }\end{array}$ & $\begin{array}{c}\text { Sensitivity } \\
\mu \mathrm{C} / \mathrm{Gy}\end{array}$ & $\begin{array}{c}\text { Specific } \\
\text { Sensitivity } \\
\mu \mathrm{C} / \mathrm{Gy} \cdot \mathrm{mm}^{3}\end{array}$ & SNR \\
\hline SC-C/Ni & 50 & $2.12 \times 10^{-7}$ & $1.1 \times 10^{-9}$ & $2.9 \times 10^{-11}$ & $1.06 \times 10^{-8}$ & $\sim 20$ & 7.8 & 4.87 & $7.2 \times 10^{3}$ \\
\hline
\end{tabular}

Table (1): The gain factor, sensitivity, specific sensitivity and SNR of SC-C/Ni diamond sample.

A very high photoconductive gain was obtained in previous reports $[13,6,8]$ with values from $6 \times 10^{4}$ to $10^{5}$.

Using the data obtained from table (1) to measure both the average currents under irradiation and dark current a significant high signal to noise ratio (SNR) was observed for the SC-C/Ni sample. The SNR is satisfactory as per the recommendations of the IAEA (>1000). The SC-C/Ni SNR value of $7.2 \times 10^{3}$ is more than two times greater than the Poly-C/Ni $\left(\sim 3.3 \times 10^{3}\right)$ value. The SNR of the SC-C/Ni is between the value reported by Galbiati et al. [9] who found a SNR of $3.3 \times 10^{4}$ for SC CVD diamond crystal with argon magnetron sputtered DLC/Pt/Au electrodes on both sides under irradiation by Co-60 $\gamma$-rays at bias of $100 \mathrm{~V}$ and a SNR of 776 found in another report [26] for $\mathrm{SC}$ diamond with an electrode of $\mathrm{Ni}$ on one side and $\mathrm{Au}$ on the other side.

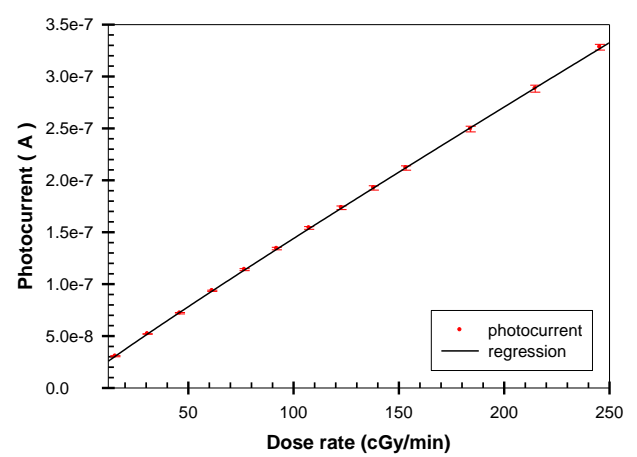

Figure (3): Dose rate dependence of diamond sample at different dose rates ranging from 0.1 to $2.5 \mathrm{~Gy} / \mathrm{min}$.

\section{Conclusions}

The target of this work was to progress the development of a near-tissue equivalent dosimeter for X-rays based on free standing SC diamond with $\mathrm{C} / \mathrm{Ni}$ electrodes deposited using PLD. The linear current response with dose rate improved with a $\Delta$ value of 0.86 to 0.97 when compared to polycrystalline diamond. This suggests that $\Delta$ changes according to the nature of detector material.

The sensitivity and specific sensitivity of SC-C/Ni sample showed a value of $\sim 7.8 \mu \mathrm{C} / \mathrm{Gy}$ and $4.87 \mu \mathrm{C} / \mathrm{Gy} . \mathrm{mm}^{3}$, respectively. The higher sensitivity in $\mathrm{SC}-\mathrm{C} / \mathrm{Ni}$ compared to Poly-C/Ni is believed to be due to the lower density 
defects than in the polycrystalline sample and the higher $C C E$ in the single crystal sample (SC-C/Ni) as seen in the alpha spectroscopy result. The SC-C/Ni showed a superior electron energy resolution of $\sim 3 \%$ to $5.48 \mathrm{MeV} \alpha$ source than the corresponding value of hole drift which was $\sim 8.5 \%$. The observations suggest that the electron signals properties for $\mathrm{SC}-\mathrm{C} / \mathrm{Ni}$ are superior to those of hole transport, due to trapping effects at the bulk material/electrode interface that predominantly affect one carrier type. This preferential trapping of one charge carrier type may also explain the occurrence of photoconductive gain in continuous current measurements.

The gain value of SC-C/Ni ( 20) device is approximately 450 times greater than the typical value of Poly-C/Ni. The SNR was satisfactory as per the recommendations of the IAEA $(>1000)$ i.e. $\sim 7.3 \times 10^{3}$. Hence, further developments of devices fabricated with PLD deposited DLC electrodes have the potential to operate at medical dosimeters.

\section{References}

1. De Angelis, C., Bucciolini, M., Casati, M., Bruzzi, M., Lagomarsino, S., Sciortino, S., Lovik, I., Onori, S., Improvements in CVD diamond properties for radiotherapy dosimetry. Radiation Protection Dosimetry 2006. 120(1-4): p. 38-42

2. Guerrero, M.J., Tromson, D., Descamps, C., Bergonzo, P., Recent improvements on the use of CVD diamond ionisation chambers for radiotherapy applications. Diamond and Related Materials, 2006. 15(4-8): p. 811-814.

3. Kuzmany, H., Pfeiffer, R., Salk, N., Günther, B., The mystery of the $1140 \mathrm{~cm}^{-1}$ Raman line in nanocrystalline diamond films. Carbon, 2004. 42(5-6): p. 911-917.

4. Bergonzo, P., Brambilla, A., Tromson, D., Mer, C., Guizard, B., Marshall, R. D., Foulon, F., CVD diamond for nuclear detection applications. Nuclear Instruments and Methods in Physics Research Section A: Accelerators, Spectrometers, Detectors and Associated Equipment, 2002. 476(3): p. 694-700.

5. $\quad$ Fidanzio, A., Azario, L., Viola, P., Ascarelli, P., Cappelli, E., Conte, G., Piermattei, A., Photon and electron beam dosimetry with a CVD diamond detector. Nuclear Instruments and Methods in Physics Research Section A: Accelerators, Spectrometers, Detectors and Associated Equipment, 2004. 524(1-3): p. 115-123.

6. Gutiérrez, A., Díaz, J., López, M. F., X-ray absorption spectroscopy study of pulsed-laser-evaporated amorphous carbon films. Applied Physics A: Material Science \& Processing, 1995. 61(2): p. 111-114.

7. Pomorski, M.B., E., Caragheorgheopol, A.,Ciobanu, M., Kiš, M., Martemiyanov, A., Nebel, C., Moritz, P., Development of single-crystal CVD-diamond detectors for spectroscopy and timing. physica status solidi (a), 2006. 203(12): p. 3152-3160.

8. Pomorski, M., Berdermann, E., de Boer, W., Furgeri, A., Sander, C., Morse, J., Charge transport properties of single crystal CVD-diamond particle detectors. Diamond and Related Materials, 2007. 16(4-7): p. 1066-1069.

9. Pernegger, H., Roe, S., Weilhammer, P., Eremin, V., Frais-Kölbl, H., Griesmayer, E., Kagan, H., Schnetzer, S., Stone, R., Trischuk, W., Twitchen, D., Whitehead, A., Charge-carrier properties in synthetic single-crystal diamond measured with the transient-current technique. Journal of Applied Physics, 2005. 97(7): p. 073704073704-9.

10. Schirru, F., Chokheli, D. andKiš, M., Thin single crystal diamond detectors for alpha particle detection. Diamond and Related Materials, 2014. 49: p. 96-102.

11. Jayawardena, K.D.G.I., Tan, Y.Y., Fryar, J., Shiozawa, H., Silva, S.R.P., Henley, S.J., Fuge, G.M., Truscott, B. S. and Ashfold, M. N. R., Highly conductive nanoclustered carbon:nickel films grown by pulsed laser deposition, Carbon 201149 (12): pp. 3781-3788.

12. Abdel-Rahman, M.A.E., Lohstroh, A., Jayawardena, I., Henley, S.J., The X-ray detection performance of polycrystalline CVD diamond with pulsed laser deposited carbon electrodes. Diamond and Related Materials, 2012. 22: p. 70-76.

13. Lohstroh, A., Sellin, P.J., Boroumand, F. Morse, J., High gain observed in X-ray induced currents in synthetic single crystal diamonds. physica status solidi (a), 2007. 204(9): p. 3011-3016.

14. Abdel-Rahman, M.A.E. The influence of electrode fabrication on induced X-ray photocurrents in CVD diamond radiation detectors, PhD thesis, University of Surrey, UK 2011.

15. National Institute of Standards and Technology, Physical Meas. Laboratory, https://physics.nist.gov/PhysRefData/Xcom/Text/intro.html (accessed 29/05/2019)

16. Schirru, F., Kupriyanov, I., Marczewska, B., Nowak, T., Radiation detector performances of nitrogen doped HPHT diamond films. physica status solidi (a), 2008. 205(9): p. 2216-2220.

17. Fowler, J.F., Solid state electrical conduction dosimeters. edited by Attix, Roesch, and Tochlin, Radiation Dosimetry II (Academic Press Inc., 1966), Chapter 14, p. 308. 
18. Biersack, J.P., Haggmark, L. G., A Monte Carlo computer program for the transport of energetic ions in amorphous targets. Nuclear Instruments and Methods, 1980. 174(1-2): p. 257-269.

19. Delacroix, D., Guerre, J. P., Leblanc, P., Hickman, C. Radionucleide and Radiation Protection Data Handbook 2002, $2^{\text {nd }}$ Ed., Radiation Protection Dosimetry, 98(1), Nuclear Technology Publishing 2002.

20. Hecht K., Zum Mechanismus des lichtelektrischen Primärstroms in isolierenden Kristallen. Z. Phys., 1932.77: p. 235

21. Galbiati, A., Lynn, S., Oliver, K., Schirru, F., Nowak, T., Marczewska, B., Duenas, J.A., Berjillos, R., Martel, I., Lavergne, L.. Performance of Monocrystalline Diamond Radiation Detectors Fabricated Using TiW, Cr/Au and a Novel Ohmic DLC/Pt/Au Electrical Contact. IEEE Transactions on Nuclear Science, 2009. 56(4): p. 1863-1874.

22. Descamps, C., Tromson, D., Tranchant, N., Isambert, A., Bridier, A., De Angelis, C., Onori, S., Bucciolini, M., Bergonzo, P., Clinical studies of optimised single crystal and polycrystalline diamonds for radiotherapy dosimetry. Radiation Measurements, 2008. 43(2-6): p. 933-938.

23. De Angelis, C.O., S., Pacilio, M., Cirrone, G. A. P., Cuttone, G., Raffaele, L., Bucciolini, M., Mazzocchi, S., An investigation of the operating characteristics of two PTW diamond detectors in photon and electron beams. Medical Physics, 2002. 29(2): p. 248-254.

24. Hugtenburg, R.P., Johnston, K., Chalmers, G.J., Beddoe, A. H., , Application of diamond detectors to the dosimetry of 45 and $100 \mathrm{kVp}$ therapy beams. Physics in Medicine \& Biology, 2001. 46 (9): pp. 2489-2501.

25. Ade, N., andNam, T.L., The influence of defect levels on the dose rate dependence of synthetic diamond detectors of various types on exposures to high-energy radiotherapy beams. Radiation Physics and Chemistry, 2015. 108: p. 65-73.

26. Cirrone, G.A.P., Cuttone, G., Lo Nigro, S., Mongelli, V., Raffaele, L., Sabini, M. G., Dosimetric characterization of CVD diamonds in photon, electron and proton beams. Nuclear Physics B - Proceedings Supplements, 2006. 150: p. 330-333.

27. Ade, N., An investigation of the role of defect levels on the radiation response of synthetic diamond crystals when used as sensors for the detection of mammography X-rays. Applied Radiation and Isotopes, 2017. 127 p. 237-244.

28. McKeag, R.D., Marshall, R.D., Baral, B., Chan, S.S.M., Jackman, R.B., Photoconductive properties of thin film diamond. Diamond and Related Materials, 1997. 6(2-4): p. 374-380.

29. Galbiati, A.B., GB), CONTACTS ON DIAMOND. 2010: United States.

30. Tranchant, N., Tromson, D., Descamps, C., Isambert, A., Hamrita, H., Bergonzo, P., Nesladek, M., High mobility single crystal diamond detectors for dosimetry: Application to radiotherapy. Diamond and Related Materials, 2008. 17(7-10): p. 1297-1301. 